\title{
Publisher Correction: The UMBRELLA SIOP-RTSG 2016 Wilms tumour pathology and molecular biology protocol
}

Gordan M. Vujanić, Manfred Gessler(D, Ariadne H. A. G. Ooms, Paola Collini, Aurore Coulomb-l'Hermine, Ellen D'Hooghe, Ronald R. de Krijger, Daniela Perotti, Kathy Pritchard-Jones (D), Christian Vokuhl, Marry M. van den Heuvel-Eibrink, Norbert Graf(D) and on behalf of the International Society of Paediatric Oncology-Renal Tumour Study Group (SIOP-RTSG)

Nature Reviews Urology (2018) https://doi.org/10.1038/s41585-018-0100-3

Published online 11 October 2018

In the version of this article initially published online it was not open access, this has now been corrected.

https://doi.org/10.1038/s41585-019-0191-5 I Published online 1 May 2019 